\title{
Determination of the loads on the rollers of the tubular belt conveyor
}

\author{
Andrii Smirnov ${ }^{1, *}$, and Tamara Mishchenko ${ }^{1}$ \\ ${ }^{1}$ Institute of Geotechnical Mechanics named by N. Poljakov of National Academy of Sciences of \\ Ukraine, 49005, Dnipro, Simferopolska Str., 2a, Ukraine
}

\begin{abstract}
The article presents methods for determining loads on rollers of a six-roll support of a tubular belt conveyor, based on the equilibrium laws of elastic cylindrical plates and shells filled with a freight. In this case, the forces acting on the rollers of the tubular conveyor are the sum of the forces caused by the weight and expansion of the bulk load, the weight and bend of the belt, as well as the inertial forces associated with the curvature and the deflection of the belt under the weight of the bulk load. The dynamic forces acting on the rollers as the belt moves are determined from the equation of the oscillations of a heavy flexible string using the Ritz method. As a result of the research, analytical dependences of the forces acting on the rollers of the six-roller support of the tubular conveyor on the parameters of the conveyor, the belt, the properties of the bulk load and the radius of curvature of the conveyor are obtained, and an assessment of the component these forces is given. The results can be used to determine the parameters of the tubular conveyors rollers and increase the service life of the rollers.
\end{abstract}

\section{Introduction}

With the increasing demands for environmental protection, tubular belt conveyors are widely used in the transportation of environmentally hazardous bulk loads.

The main advantages of tubular belt conveyors, as compared to belt conveyors with a grooved belt, are the absence of contact with the environment, as well as the ability to transport bulk load along curved paths without overload devised.

The operating experience of the tubular belt conveyors showed that, unlike the belt conveyor with a grooved belt, the rollers of the tubular conveyor are affected by additional forces due to the load spread and the inertia forces associated with the framework curvature and bend of the belt from the weight of the load. These forces acting on the rollers of the tubular conveyor, significantly reduce their life.

Therefore, to increase the life of the roller of the tubular conveyor, it is necessary, first of all, to determine the magnitudes of these forces. In the works of V.G. Dmitriev, V.I. Galkin, N.V. Sergeeva, A.P. Egorov, N.Yu. Ivanov, the calculation of loads on sixroller rollers supports of a tubular conveyor is given. However, in these studies, the obtained values of the loads on the rollers do not quite correspond to the real loads. This is

\footnotetext{
*Corresponding author: sm.contur24@gmail.com
} 
due to the fact that the methods for determining these loads are not based on the equilibrium laws of elastic cylindrical shells. In addition, in these works, the dependences of the forces acting on the rollers of tubular conveyors on the parameters of the conveyor, the belt and the properties of the transported load are not investigated.

\section{Methods}

In this paper, based on the equilibrium equations of a cylindrical elastic shell filled with a bulk load, the forces acting on the rollers of a six-roll support of a tubular conveyor are determined by the weight and spread of the bulk load, the weight and bend of the belt in its cross section, and also by the inertial forces due to curvature traces and belt deflection arising under the action of gravity of the transported load. In this case, the theory of equilibrium of an elastic cylindrical shell and the theory of the limit equilibrium of freight are used.

At first to consider the case, not taking into account dynamic efforts operating on the rollers of tubular conveyer at motion of belt with a load by the roller supports of conveyer.

According to [1-4], the force acting on the roller supports rollers of the straight section of the tubular belt conveyor consists of the sum of the forces from the weight and the forces of the expansion of the bulk load, the weight of the belt and the bending of the conveyor belt, i.e.

$$
P_{i}=P_{L d i}+P_{l i}+P_{b n i}(i=1, \ldots, 6)
$$

where $P_{i}$ is force acting on the $i$-th roller support roller of the tubular conveyor (Fig. 1), $\mathrm{N} ; P_{L d i}$ is force acting on the $i$-th roller support roller of the tubular conveyor on the weight and expansion of the bulk load, $\mathrm{N} ; P_{l i}$ is force acting on the $i$-th roller support roller of the tubular conveyor on the weight of the belt (see Fig. 1), N; and $P_{b n i}$ is force acting on the $i$-th roller of the tubular conveyor from the bending of the belt, $\mathrm{N}$.

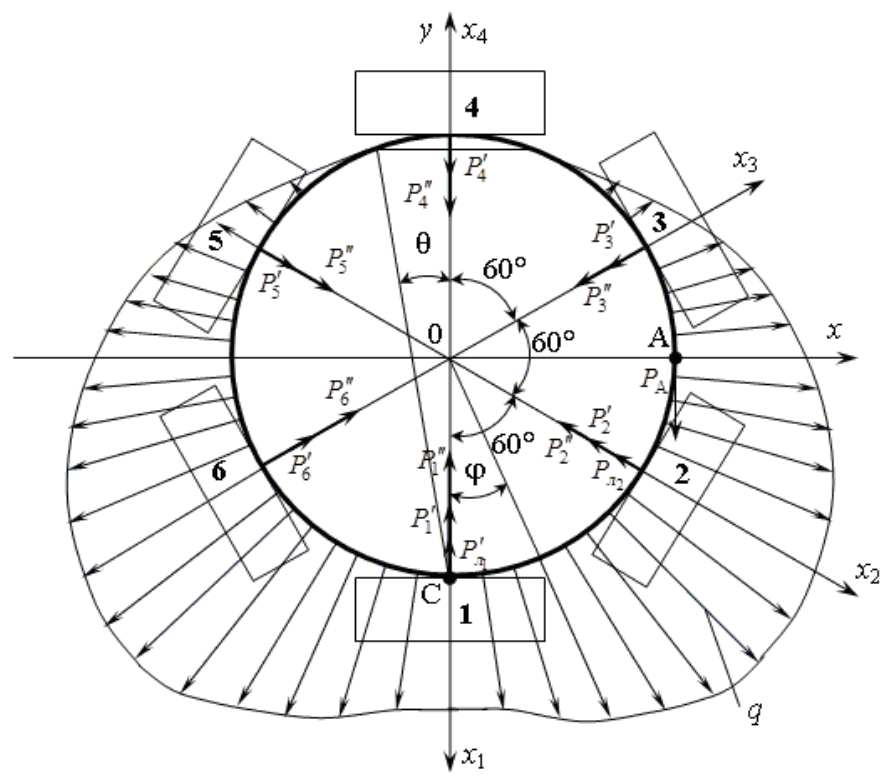

Fig. 1. The design scheme of the forces acting on the rollers of the tubular conveyor.

To determine the forces acting on the rollers of the tubular conveyor on the weight and 
expansion of the bulk load, assume that the tubular conveyor belt is an open cylindrical elastic shell of radius $\mathrm{R}$, completely filled with bulk load and moving along the roller supports with six rollers. The average value of the normal distributed load on the belt is determined by the formula:

$$
q=\frac{q_{a}+q_{p}}{2}
$$

where $q_{a}$ is active normal distributed load on the conveyor belt associated with its compression, $\mathrm{N} / \mathrm{m}^{2}$; and $q_{p}$ is passive normal distributed load on the conveyor belt associated with its expansion, $\mathrm{N} / \mathrm{m}^{2}$.

At the same time, active and passive normal distributed loads are respectively equal to [5]:

$$
\begin{aligned}
& q_{a}=R \gamma\left(\cos ^{2} \varphi+m \sin ^{2} \varphi\right)(\cos 2 \theta+\cos \varphi) ; \\
& q_{p}=R \gamma\left(\cos ^{2} \varphi+\frac{\sin ^{2} \varphi}{m}\right)(\cos 2 \theta+\cos \varphi),
\end{aligned}
$$

where $m$ is coefficient of mobility of bulk load, $m=1+2 f^{2}-2 f \cdot \sqrt{1+f^{2}} ; f$ is coefficient of internal friction of the bulk material, [6]; $\gamma$ is volume weight of the transported load, $\mathrm{N} / \mathrm{m}^{3} ; \varphi$ is current angular coordinate in the cross section of the tubular conveyor belt, rad; and $\theta$ is angle that determines the degree of filling the bulk material of the cross-section of the belt contour, rad.

Substituting equalities (3) into (2), after transformation we get:

$$
q(\varphi)=\left\{\begin{array}{l}
0,5 R \gamma\left(\cos 2 \theta_{1}+\cos \varphi\right)\left(2 \cos ^{2} \varphi+\frac{m^{2}+1}{m} \sin ^{2} \varphi\right), 0 \leq \varphi \leq \varphi_{1} ; \\
0,5 R \gamma\left(\cos 2 \theta_{2}+\cos \varphi\right)\left(2 \cos ^{2} \varphi+\frac{m^{2}+1}{m} \sin ^{2} \varphi\right), \varphi_{2} \leq \varphi \leq 0,
\end{array}\right.
$$

where $\left.\varphi_{1}=\pi-2 \theta_{1} ; \varphi_{2}=-\left(\pi-2 \theta_{2}\right)\right) ; \theta_{1}$ and $\theta_{2}$ are degree of filling of the left and right sides of the cross section of the belt, rad.

In the case of a full loading of bulk load of tubular belt $\left(\theta_{1}=\theta_{2}=0\right)$ equality (4) takes the form:

$$
q(\varphi)=\left\{\begin{array}{l}
0,5 R \gamma(1+\cos \varphi)\left(2 \cos ^{2} \varphi+\frac{m^{2}+1}{m} \sin ^{2} \varphi\right), 0 \leq \varphi \leq \pi \\
0,5 R \gamma(1+\cos \varphi)\left(2 \cos ^{2} \varphi+\frac{m^{2}+1}{m} \sin ^{2} \varphi\right),-\pi \leq \varphi \leq 0 .
\end{array}\right.
$$

To determine the forces acting on the rollers from the weight and expansion of the bulk load, select between the roller parts three parts of the belt, limited by the contact points of the belt with the 1st and 2nd rollers, the points of contact with the 2 nd and 3rd rollers, and with $3 \mathrm{rd}$ and 4 th rollers.

Suppose that there are no friction forces between the belt and the rollers. In this case, the normal forces acting on the rollers from the side of the belt and the load take on maximum values.

Let us sum up the projections of all the forces on the axis $0 x_{1}, 0 x_{2}, 0 x_{3}, 0 x_{4}$, acting on each selected part of the belt, and equate these sums to zero. 
As a result, we get:

$$
\begin{aligned}
& P_{1}^{\prime}+\frac{P_{2}^{\prime \prime}}{2}=l_{r} \int_{0}^{60^{\circ}} q R \cos \varphi d \varphi ; \\
& P_{2}^{\prime \prime}+\frac{P_{1}^{\prime}}{2}=l_{r} \int_{0}^{60^{\circ}} q R \cos \varphi d \varphi ; \\
& P_{2}^{\prime}+\frac{1}{2} P_{3}^{\prime}=l_{r} \int_{60^{\circ}}^{120^{\circ}} q R \cos \left(\varphi-60^{\circ}\right) d \varphi ; \\
& P_{3}^{\prime \prime}+\frac{1}{2} P_{2}^{\prime}=l_{r} \int_{60^{\circ}}^{120^{\circ}} q R \cos \left(120^{\circ}-\varphi\right) d \varphi ; \\
& \frac{1}{2} P_{4}^{\prime}+P_{3}^{\prime}=l_{r} \int_{1}^{\varphi_{1}} q R \cos \left(\varphi-120^{\circ}\right) d \varphi ; \\
& P_{4}^{\prime}+\frac{1}{2} P_{3}^{\prime}=l_{r} \int_{120^{\circ}}^{\varphi_{1}} q R \cos \left(180^{\circ}-\varphi\right) d \varphi
\end{aligned}
$$

where $\varphi_{1}=180^{\circ}-2 \theta$.

Here $P^{\prime}{ }_{1}$ is the force acting on the 1 st dedicated part of the belt from the 1 st roller, N; $P_{2}^{\prime}, P^{\prime \prime}{ }_{2}$ is the forces acting on the 1 st and 2 nd allocated parts of the belt from the 2 nd roller, respectively, $\mathrm{N} ; P_{3}^{\prime}, P^{\prime \prime}{ }_{3}$ is the forces acting on the 2 nd and $3 \mathrm{rd}$ allocated parts of the belt from the 3 rd roller, respectively, $\mathrm{N} ; P^{\prime}{ }_{4}$ is the force acting on the 3 rd dedicated part of the belt from the 4th roller, $\mathrm{N}$; and $l_{r}$ is distance between the rollers, $\mathrm{m}$.

The forces acting on the 1st, 2nd, 3rd and 4th rollers of the roller carriers due to the forces of weight and expansion of the bulk load are defined by the formulas:

$$
P_{L d 1}=2 P_{1}^{\prime} ; \quad P_{L d 2}=P_{2}^{\prime}+P_{2}^{\prime \prime} ; \quad P_{L d 3}=P_{3}^{\prime}+P_{3}^{\prime \prime} ; \quad P_{L d 4}=2 P_{4}^{\prime} .
$$

Solving the system of equations (6)-(11) with respect to the unknowns $P_{1}^{\prime}, P_{2}^{\prime}, P^{\prime \prime}{ }_{2}, P_{3}^{\prime}$, $P^{\prime \prime}{ }_{3}, P^{\prime}{ }_{4}$ and substituting them into equations (12), we obtain the forces acting on the rollers equal to:

$$
\begin{gathered}
P_{L d 1}=\frac{8}{3} l_{r} \int_{0}^{60^{\circ}} q R \cos \varphi d \varphi-\frac{4}{3} l_{r} \int_{0}^{60^{\circ}} q R \cos \left(60^{\circ}-\varphi\right) d \varphi \\
P_{L d 2}=\frac{4}{3} l_{r} \int_{0}^{120^{\circ}} q R \cos \left(\varphi-60^{\circ}\right) d \varphi-\frac{2}{3} l_{r} \int_{60^{\circ}}^{120^{\circ}} q R \cos \left(120^{\circ}-\varphi\right) d \varphi-\frac{2}{3} l_{r} \int_{0}^{60^{\circ}} q R \cos \varphi d \varphi \\
P_{L d 3}=\frac{4}{3} l_{r} \int_{60^{\circ}}^{120^{\circ}} q R \cos \left(120^{\circ}-\varphi\right) d \varphi-\frac{2}{3} l_{r} \int_{60^{\circ}}^{120^{\circ}} q R \cos \left(\varphi-60^{\circ}\right) d \varphi+ \\
+\frac{4}{3} l_{r} \int_{120^{\circ}}^{\varphi_{1}} q R \cos \left(\varphi-120^{\circ}\right) d \varphi-\frac{2}{3} l_{r} \int_{120^{\circ}}^{\varphi_{1}} q R \cos \left(180^{\circ}-\varphi\right) d \varphi \\
P_{L d 4}=\frac{8}{3} l_{r} \int_{120^{\circ}}^{\varphi} q R \cos \left(180^{\circ}-\varphi\right) d \varphi-\frac{4}{3} l_{r} \int_{120^{\circ}}^{\varphi 1} q R \cos \left(\varphi-120^{\circ}\right) d \varphi
\end{gathered}
$$

where $q=0,5 l_{r} R \gamma(\cos 2 \theta+\cos \varphi)\left(2 \cos ^{2} \varphi+\frac{m^{2}+1}{m} \sin ^{2} \varphi\right) ; 0 \leq \varphi \leq \varphi_{1} ; \varphi_{1}=180^{\circ}-2 \theta ; 0 \leq \theta \leq 90^{\circ}$.

Taking the integrals in the right-hand sides of equalities (13)-(16), after the 
transformation we get:

$$
P_{L d 1}=I_{1} \gamma R^{2} l_{r} ; P_{L d 2}=I_{2} \gamma R^{2} l_{r} ; P_{L d 3}=I_{3} \gamma R^{2} l_{r} ; P_{L d 4}=I_{4} \gamma R^{2} l_{r} ; P_{L d 5}=I_{3} \gamma R^{2} l_{r} ; P_{L d 6}=I_{2} \gamma R^{2} l_{r} \text {, }
$$

where

$$
\begin{aligned}
& I_{1}=\frac{8}{3} \int_{0}^{60^{\circ}} \bar{q} \cos \varphi d \varphi-\frac{4}{3} \int_{0}^{60^{\circ}} \bar{q} \cos \left(60^{\circ}-\varphi\right) d \varphi ; \\
& I_{2}=\frac{4}{3} \int_{0}^{120^{\circ}} \bar{q} \cos \left(\varphi-60^{\circ}\right) d \varphi-\frac{2}{3} \int_{60^{\circ}}^{120^{\circ}} \bar{q} \cos \left(120^{\circ}-\varphi\right) d \varphi-\frac{2}{3} \int_{0}^{60^{\circ}} \bar{q} \cos \varphi d \varphi ; \\
& I_{3}=\frac{4}{3} \int_{60^{\circ}}^{120^{\circ}} \bar{q} \cos \left(120^{\circ}-\varphi\right) d \varphi-\frac{2}{3} \int_{60^{\circ}}^{120^{\circ}} \bar{q} \cos \left(\varphi-60^{\circ}\right) d \varphi+ \\
& +\frac{4}{3} \int_{120^{\circ}}^{\varphi_{1}} \bar{q} \cos \left(\varphi-120^{\circ}\right) d \varphi-\frac{2}{3} \int_{120^{\circ}}^{\varphi_{1}} \bar{q} \cos \left(180^{\circ}-\varphi\right) d \varphi \\
& I_{4}=\frac{8}{3} \int_{120^{\circ}}^{\varphi_{1}} \bar{q} \cos \left(180^{\circ}-\varphi\right) d \varphi-\frac{4}{3} \int_{120^{\circ}}^{\varphi_{1}} \bar{q} \cos \left(\varphi-120^{\circ}\right) d \varphi \\
& \bar{q}=0,5(\cos (2 \theta)+\cos \varphi)\left(2 \cos ^{2} \varphi+\frac{m^{2}+1}{m} \sin ^{2} \varphi\right) ; \\
& 0 \leq \varphi \leq \varphi_{1} ; \varphi_{1}=180^{\circ}-2 \theta ; 0 \leq \theta \leq 90^{\circ} \text {. }
\end{aligned}
$$

From formulas (17) it follows that the magnitude of the forces acting on the six-roller roller supports of the tubular conveyor, caused by the weight and spread of the bulk load, is proportional to the square of the radius of the tubular belt and is directly proportional to the specific weight of the bulk load and the distance between the roller supports.

Table 1 shows the values of the coefficients $I_{1}, I_{2}, I_{3}, I_{4}$, for $\theta=0$ and various values of the friction coefficient of the bulk load $f$.

Table 1. The values of the coefficients $I_{1}, I_{2}, I_{3}, I_{4}$ with $\theta=0$ and different values of the friction coefficient of the bulk load $f$.

\begin{tabular}{|c|c|c|c|c|c|c|c|c|c|c|}
\hline$f$ & 0.1 & 0.2 & 0.3 & 0.4 & 0.5 & 0.6 & 0.7 & 0.8 & 0.9 & 1.0 \\
\hline$I_{1}$ & 2.209 & 2.230 & 2.264 & 2.313 & 2.375 & 2.451 & 2.541 & 2.645 & 2.762 & 2.894 \\
\hline$I_{2}$ & 1.698 & 1.759 & 1.860 & 2.001 & 2.183 & 2.405 & 2.667 & 2.970 & 3.313 & 3.696 \\
\hline$I_{3}$ & 0.642 & 0.674 & 0.727 & 0.801 & 0.896 & 1.013 & 1.151 & 1.310 & 1.491 & 1.692 \\
\hline$I_{4}$ & 0.108 & 0.111 & 0.115 & 0.120 & 0.127 & 0.136 & 0.146 & 0.157 & 0.171 & 0.185 \\
\hline
\end{tabular}

When the belt is fully loaded, i.e. when $\theta=0^{\circ}$ and $f=0.7(m=0.271)$, the values of the integrals $I_{1}, I_{2}, I_{3}, I_{4}$ are:

$$
I_{1}=2.541 ; \quad I_{2}=2.667 ; \quad I_{3}=1.151 ; \quad I_{4}=0.146 .
$$

We now determine the forces acting on the roller support rollers of the tubular conveyor on the weight of the belt. To do this, we select a section of the AC belt between the rollersupports without a load (see Fig. 1). The following forces will act on this section of the conveyor belt: $P_{A}$ is force from the upper part of the conveyor belt; $P_{b 1}^{\prime}$ is reaction force from the bottom roller 1 and directed vertically upwards; $P^{\prime}{ }_{b 2}$ is the reaction force from the side of the lower side roller 2 and directed to the center of the cylinder, as well as the 
distributed weight forces of the selected section of the belt, directed vertically downwards. It is assumed that the friction forces between the belt and the rollers are absent. In this case, the normal forces acting on the rollers from the side of the belt and the load take on maximum values.

In addition, we assume that the forces acting on the upper, central and lateral upper rollers of the belt weight are zero.

Let us write the equilibrium equations of these forces acting on the selected section of the $\mathrm{AC}$ belt, in projections onto the axis $0 \mathrm{y}$ and the moments of these forces relative to point C. As a result, we obtain a system of equations for forces $P_{b 1}^{\prime}$ and $P_{b 2}^{\prime}$ :

$$
\left\{\begin{array}{l}
P_{b 1}^{\prime}+P_{b 2} \cos 60^{\circ}-P_{A}-\frac{q_{b} l_{r}}{4}=0 ; \\
P_{A} R-P_{b 2} R \cos 60^{\circ}+l_{r} \int_{0}^{90^{\circ}} q_{b}^{\prime} R^{2} \sin \varphi d \varphi=0 .
\end{array}\right.
$$

Here $q_{b}^{\prime}$ is the distributed load of the belt weight, determined by the formula

$$
q_{b}^{\prime}=\frac{q_{b}}{B}
$$

where $q_{b}$ is linear weight of the belt, $\mathrm{N} / \mathrm{m}$; and $B$ is width of the belt, $\mathrm{m}$

Taking the integral in the second equation of system (19) and solving it with respect to $P^{\prime}{ }_{b 1}$ and $P_{b 2}$, we get

$$
\left\{\begin{array}{l}
P_{b 1}^{\prime}=P_{A}+\frac{q_{b} l_{r}}{4}-\frac{P_{b 2}}{2} \\
P_{b 2}=\frac{2}{\sqrt{3}}\left(P_{A}+q_{b}^{\prime} l_{r} R\right)
\end{array}\right.
$$

The force $P_{A}$ is equal to the fourth part of the belt weight on the section between the roller supports, i.e.

$$
P_{A}=\frac{q_{b} l_{r}}{4} .
$$

Substituting (20) and (22) into (21), after the conversion we get:

$$
\begin{aligned}
& P_{b 1}^{\prime}=\left(\frac{1}{2}-\frac{1}{4 \sqrt{3}}-\frac{1}{\sqrt{3}} \frac{R}{B}\right) q_{b} l_{r} ; \\
& P_{b 2}=\frac{2}{\sqrt{3}}\left(\frac{1}{4}+\frac{R}{B}\right) q_{b} l_{r} .
\end{aligned}
$$

The force acting on the lower roller 1 from the entire belt is equal to

$$
P_{b 1}=2 P_{b 1}^{\prime}
$$

Substituting the last equality in (22), we get 


$$
P_{b 1}=\left(1-\frac{1}{2 \sqrt{3}}-\frac{2}{\sqrt{3}} \frac{R}{B}\right) q_{b} l_{r} .
$$

The width of the belt of the tubular conveyor can be approximately determined by the formula

$$
B=2 \pi R
$$

Substituting (26) into (24) and (25) taking into account the symmetry of the forces acting on the left side roller of the roller support, after calculation we get:

$$
P_{b 1}=0.528 q_{b} l_{r} ; P_{b 2}=0.473 q_{b} l_{r} ; P_{b 3}=0 ; P_{b 4}=0 ; P_{b 5}=0 ; P_{b 6}=0.473 q_{b} l_{r} .
$$

From (27) it follows that the forces acting on the rollers of the tubular conveyor, due to the weight of the belt, are directly proportional to the linear load and the distance between the rollers.

We now determine the forces $P_{b n i}$ acting on the rollers from the bending of the belt.

The forces acting on the rollers from the bending of the belt can be represented as the sum of the forces from the preliminary bending of the belt without taking into account the load and the forces from the bending of the belt under the action of the forces of spreading the bulk load. The deflection of the belt from the preliminary bending of the belt is much greater than the amount of deflection of the belt from the forces of expansion of the bulk load. Therefore, the forces acting on the rollers from the bending of the belt, due to the forces of expansion of the bulk load, are much less than the forces acting on the rollers from the preliminary bending of the belt. Since the bending stiffness of the belt is small, the forces acting on the rollers from the preliminary bending of the belt are small and can be neglected. Therefore, we define only the forces acting on the rollers from the preliminary bending of the belt.

Imagine the belt as an elastic infinitely long cylindrical plate, on which a constant bending moment acts. Select the part of the tape between the points of contact with the third and fourth rollers, i.e. part 3 (see Fig. 1).

According to [7], we write the expression to determine the magnitude of the bending moment in the selected 3rd part of the tape in the form

$$
M_{b n}=\frac{D l_{r}}{R},
$$

where $M_{b n}$ is moment of force acting on the belt from the 4th roller (upper) side to the 3rd dedicated part of the belt relative to the point of contact of the 3rd roller (upper side roller) with the belt, $\mathrm{N} \cdot \mathrm{m}$; and $D$ is bending stiffness of a cylindrical plate, $\mathrm{N} \cdot \mathrm{m}$.

Bending stiffness of a cylindrical plate according to [7] is determined by the formula

$$
D=\frac{E_{b} h_{b}^{3}}{12\left(1-v^{2}\right)},
$$

where $E_{b}$ is modulus of elasticity of the belt in the longitudinal direction of the belt, $\mathrm{N} / \mathrm{m}^{2} ; h_{b}$ is belt thickness, $\mathrm{m}$; and $v$ is Poisson's coefficient of the belt.

The bending moment of the $M_{b n}$ at the point $\mathrm{C}$ of the contact of the belt with the lower roller (see Fig. 1) is equal to the sum of the torques acting on the belt at the contact points of the second, third and fourth rollers under the assumption that there is no load in the tubular conveyor belt: 


$$
M_{b n}=P_{b n 2} d_{2}+P_{b n 3} d_{3}+P_{b n 4} d_{4},
$$

where $d_{2}, d_{3}, d_{4}$ are the values of perpendiculars lowered from point $C$ to the direction of the force vectors $P_{b n 2}, P_{b n 3}, P_{b n 4}$.

According to Figure 1 values $d_{2}, d_{3}, d_{4}$ are respectively equal to:

$$
d_{2}=d_{3}=R \sin 60^{\circ}=\frac{\sqrt{3}}{2} R ; \quad d_{4}=0 .
$$

Since the bending stiffness of the belt is constant, due to the symmetry for a cylindrical belt, the forces acting on the rollers from the bending of the belt are equal

$$
P_{b n_{1}}=P_{b n 2}=P_{b n_{3}}=P_{b n_{4}}=P_{b n}
$$

Substituting (31) into (30) in view of the last equality, we get

$$
M_{b n}=\sqrt{3} R P_{b n} .
$$

From the last equality, taking into account (28), we determine the forces acting on the rollers of the tubular conveyor from the bending of the belt:

$$
P_{b n}=\frac{D l_{r}}{\sqrt{3} R^{2}} .
$$

Substituting (17), (27) and (33) into (1), we finally obtain the forces acting on the rollers of the six-roll support of the tubular conveyor, taking into account the belt weight:

$$
\begin{aligned}
& P_{1}=I_{1} \gamma R^{2} l_{r}+0.528 q_{b} l_{r}+\frac{D l_{r}}{\sqrt{3} R^{2}} ; \quad P_{2}=I_{2} \gamma R^{2} l_{r}+0.473 q_{b} l_{r}+\frac{D l_{r}}{\sqrt{3} R^{2}} \\
& P_{3}=I_{3} \gamma R^{2} l_{r}+\frac{D l_{r}}{\sqrt{3} R^{2}} ; \quad P_{4}=I_{4} \gamma R^{2} l_{r}+\frac{D l_{r}}{\sqrt{3} R^{2}} ; \quad P_{5}=I_{3} \gamma R^{2} l_{r}+\frac{D l_{r}}{\sqrt{3} R^{2}} \\
& P_{6}=I_{2} \gamma R^{2} l_{r}+0.473 q_{b} l_{r}+\frac{D l_{r}}{\sqrt{3} R^{2}}
\end{aligned}
$$

For comparison, we define the total force acting on the roller from the weight of the load and belt, according to the formula

$$
P=\pi \gamma R^{2} l_{r}+q_{b} l_{r}=3,14 \gamma R^{2} l_{r}+q_{b} l_{r} .
$$

\section{Result and discussion}

Calculations showed that the forces acting on the lower central and lower side rollers of the six-roll support of the tubular conveyor approximately coincide with the total force acting on the roller supports on the weight of the load and the belt in the area between the roller supports. At the same time, for a conveyor belt with a grooved belt, the force acting on the lower roller is 0.7 of the total force acting on the roller support on the weight of the load and belt in the area between the roller supports.

In addition, the calculations showed that the forces acting on the rollers of the six-roller support of the tubular conveyor, caused by the belt weight, do not exceed $11 \%$ of the total 
amount of weight forces and expansion of the bulk load, bending forces and belt weight.

In the case of a curved section of a tubular belt conveyor, on rollers, in addition to the forces from the weight and the forces spreading the bulk load, the weight of the belt and from the bending of the belt of the conveyor, the forces from tension of the belt the total force acting on the rollers is determined by the formula

$$
P_{i}^{\prime}=P_{L d i}^{\prime}+P_{b i}^{\prime}+P_{b n i}^{\prime}+P_{t}(i=1, \ldots, 6)
$$

where $P^{\prime}{ }_{i}$ is force acting on the $i$-th roller supports roller of the curvilinear section of the tubular conveyor (see Fig. 1), $\mathrm{N} ; P_{L d i}^{\prime}$ is force acting on the $i$-th roller supports roller, curvilinear section of the tubular conveyor from the weight and spreading bulk load, $\mathrm{N} ; P^{\prime}{ }_{b i}$ is force acting on the $i$-th roller supports roller, curvilinear section of the tubular conveyor of the weight of the belt (Fig. 1), N; $P^{\prime}{ }_{b n i}$ is force acting on the $i$-th roller supports roller, curvilinear section of the tubular conveyor from the bending of the belt, $\mathrm{N}$; and $P_{t}$ is force acting on the roller support rollers, curvilinear section of the tubular conveyor from the belt tension, N.

Forces $P_{L d i}^{\prime}, P_{b i}^{\prime}, P_{b n i}^{\prime}$ are determined by the same formulas as forces $P_{L d i}, P_{b i}, P_{b n i}$, i.e. by formulas (17), (27) and (33) only instead of $l_{r}$ is substituted $l_{r}{ }_{r}$.

The force $P_{t}$ acting on the rollers from the equilibrium triangle of forces acting on the rollers from the tension of the belt and the reaction of the rollers is determined by the formula

$$
P_{t}=S_{b} \frac{l_{r}^{\prime}}{R_{c}},
$$

where $l_{r}^{\prime}$ is distance between the rollers on the curvilinear section of the tubular conveyor framework, $\mathrm{m}$; and $R_{c}$ is radius of curvature of the curvilinear section of the tubular conveyor framework, $\mathrm{m}$

When the load moves along the roller supports of the straight line section of the tubular conveyor dynamic forces occur on the rollers of the tubular conveyor due to bend of the belt under action of gravities load and belt are arised.

At the same time, when moving small-sized load in a straight line section of the conveyor framework, dynamic forces on the rollers of the six-roll support of the tubular conveyor are determined by the formulas [8]:

$$
P_{d i}=k_{d} P_{L d i}+k_{d} P_{b i}+P_{b n}(i=1, \ldots, 6),
$$

where $k_{d}$ is coefficient of dynamics when moving small-sized load on the roller conveyor, determined by the formula

$$
k_{d}=1+\frac{v_{b}^{2}}{g} \cdot \frac{q}{\left(S_{b}-\frac{v_{b}^{2}}{g} q\right)},
$$

where $v_{b}$ is belt speed, $\mathrm{m} / \mathrm{s} ; q=q_{L d}+q_{b}$ is sum of linear weights of load and belt, $\mathrm{N} / \mathrm{m}$; and $S_{b}$ is belt tension, $\mathrm{N}$.

Research have shown that the dynamic forces acting on the rollers during the movement of small-sized load along the roller supports of the straight section of the tubular conveyor are $10.3 \%$ more compared with static loads on the rollers.

When a load is moving along a curvilinear section of a tubular conveyor framework, the maximum dynamic forces on rollers support rollers bearing arise at the starting point of a 
curvilinear section of a framework are determined by the formulas [9]:

$$
P_{d i}^{\prime}=k_{d}^{\prime} P_{L d i}^{\prime}+k_{d}^{\prime} P_{b i}^{\prime}+P_{b n}^{\prime}+P_{t}(i=1, \ldots, 6),
$$

where

$$
k_{d}^{\prime}=k_{d}\left(1+\frac{v_{b}^{2}}{R_{c} g}\right)
$$

$R_{c}$ is radius of curvature at the starting point of the curvilinear section of the tubular conveyor framework, $\mathrm{m}$.

Substituting the values in the formula (39), $P_{L d i}^{\prime}, P_{b i}^{\prime}$ and $P^{\prime}{ }_{b n}$, determined by the formulas (17), (27) and (33), replacing $l_{r}$ by $l_{r}^{\prime}$, we get the dynamic forces acting on the rollers of the six-roller support when the load moves along the curvilinear section of the tubular conveyor framework.

\section{Conclusions}

1. On the basis of the equilibrium equation of a cylindrical elastic belt moving along with a bulk load on six-roller supports of a tubular conveyor, the forces acting on the rollers are determined by the weight and spreading forces of the bulk load, the weight and bending belt as well as inertial forces and belt tension.

It is established that:

a) the magnitude of the forces acting on the rollers of the six-roller support of the tubular conveyor, caused by the weight and spread of the bulk load, are proportional to the square of the radius of the tubular belt and directly proportional to the distance between the roller supports. In this case, the forces acting on the lower central and lower side rollers, due to the weight and expansion of the bulk load, are approximately equal to the sum of the weight of the bulk load and the conveyor belt in the area between the roller supports;

b) the forces acting on the rollers of the tubular conveyor, due to the bending of the belt, are directly proportional to the bending stiffness of the belt and the distance between the roller supports and inversely proportional to the square of the radius of the tubular belt;

c) the forces acting on the rollers of the tubular conveyor, due to the weight of the belt, are directly proportional to the linear weight of the load and distance between the roller supports and do not exceed $11 \%$ of the total sum of the forces of weight and expansion of the bulk load;

d) the magnitude of the force acting on the rollers of the curvilinear section of the tubular conveyor framework, due to the tension of the belt, is directly proportional to the tension of the belt and the distance between the roller supports and is inversely proportional to the radius of curvature of the curvilinear section of the framework.

2. On the basis of the developed mathematical model of the oscillations of the tubular belt during movement along the roller conveyors, the dynamic components of the forces acting on the roller supports, determined by the weight and expansion of the bulk load, as well as the weight of the belt, are determined. At the same time, the dynamic components of the forces acting on the roller supports of the tubular conveyor do not exceed $10 \%$ of the static loads on the roller supports of the tubular conveyor.

\section{References}

1. Galkin, V.I., Dmitriev, V.G. (2009). Tubular conveyers for mining industry. Gornoe 
oborudovanie i elektromechanika, 1, 39-46

2. Dmitriev, V.G., Sergeeva, N.V. (2011). Method of hauling calculation of band tubular conveyer. Gornyy informatsionno-analiticheskiy byulleten, 7, 218-228

3. Dmitriev, V.G, Egorov, A.P. (2011). Influence of speed of belt on distributing of force of resistance to motion of tubular conveyer. Gornyy informatsionno-analiticheskiy byulleten, 6, 191-198.

4. Dmitriev, V.G., Ivanov, N.Yu. (2013). Teoriya lentochnykh trubchatykh konveerov (LTK) prostranstvennoy krivolineynoy trassy. Vypusk 1. Moskva: Gornaya kniga

5. Sheshko, E. E., Gushin, V. M. (1973). Steeply inclined conveyor belt, which has form of deep troughs. Razvitie $i$ sovershenstvovanie shachtnogo $i$ kariernogo transporta. Moskva: Nedra

6. Zenkov, R.L. (1964). Mechanika nasypnych gruzov. Moscow: Mashinostroenie

7. Timoshenko, S.P., Voynovskiy-Kriger, S. (1963). Plastiny i obolochki. Moskva: Fiz.mat. Literature

8. Kiriya, R.V., Smirnov, A.N. (2016). Determination of dynamic factor when the belt with load are transported by the tubular conveyers roller supports, Geo-Technical Mechanics, 131, 144-151

9. Kiriya, R.V., Smirnov, A.N. (2018). Decision of dynamic efforts on rolikoopori at motion of ribbon and load on the curvilinear area of tubular conveyer. Geo-Technical Mechanics, 139, 152-161 